PHYSICAL REVIEW D 93, 025030 (2016)

\title{
Canonical realization of Bondi-Metzner-Sachs symmetry: Quadratic Casimir
}

\author{
Joaquim Gomis ${ }^{1, *}$ and Giorgio Longhi ${ }^{2, \dagger}$ \\ ${ }^{1}$ Departament d'Estructura i Constituents de la Matèria and Institut de Ciències del Cosmos, \\ Universitat de Barcelona, Diagonal 647, 08028 Barcelona, Spain \\ ${ }^{2}$ Dipartimento di Fisica, Universita' di Firenze, Via Giovanni Sansone 1, \\ 50019 Sesto Fiorentino (FI), Italy \\ (Received 12 August 2015; published 27 January 2016)
}

\begin{abstract}
We study the canonical realization of Bondi-Metzner-Sacks symmetry for a massive scalar field introduced by Longhi and Materassi [J. Math. Phys. 40, 480 (1999)]. We construct an invariant scalar product for the generalized momenta. As a consequence we introduce a quadratic Casimir with the supertranslations.
\end{abstract}

DOI: 10.1103/PhysRevD.93.025030

\section{MOTIVATION AND RESULTS}

Recently there has been a renewed interest in the BondiMetzner-Sacks (BMS) group [1]. The BMS invariance of the gravitational scattering [2], the relation among soft graviton theorems [3] and BMS supertranslations [4] have been shown. The relation among supertranslations, gravitational memory and soft gravitons theorems has been also studied [5]. There is also the proposal that the BMS group could be useful to understand holography in asymptotically flat space-times [6-9]. Related to these developments has been the study of asymptotic symmetries in quantum field theories; in the case of QED, see [10] for the massless case and $[11,12]$ for the massive case. A recent overview on the whole subject is given by Strominger [13] at Strings 2015. Lately Hawking put forward the idea that supertranslations could solve the information paradox problem of black holes [14].

In this work we study the canonical realization of the BMS symmetry for a free massive real scalar field in four dimensions introduced in [15]. The Poincaré generators $P^{\mu}$, $M^{\mu \nu}$ are written in terms of the Fourier modes $a(\vec{k}), a^{*}(\vec{k})$ of the plane wave expansion of the Klein-Gordon field. The momentum mass-shell condition $q^{2}-m^{2}=0$ defines a 3D spacelike hyperboloid $H_{3}^{1}$ [16]. It is useful to introduce a differential operator in this space $D=-m^{2} \Delta+3$, where $\Delta$ is the Laplace-Beltrami operator on $H_{3}^{1}$.

It happens that the four-dimensional momenta $k^{\mu}$ are zero modes of $D$; this suggests to look for the zero modes of this operator in general. These are given by an infinite set of function $w_{l, m}$ defined on $H_{3}^{1}$, unique up to rescalings, $l=0,1,2, \ldots|m| \leq l$. The explicit expression of this functions was given in [15]; see also the next section. The functions $w_{l, m}$ can be considered as a generalization of four

gomis@ecm.ub.edu

longhi@fi.infn.it momenta. This allows us to define the supertranslations for massive scalar field $P_{l, m}$ in terms of $w_{l, m}$ and the Fourier modes $a(\vec{k}), a^{*}(\vec{k})$; see the next section.

The transformation of the scalar field under space-time translations is obtained from the scalar nature of the field under Poincaré transformations. This is not the case for supertranslations. The supertranslations act on the field and their conjugated momenta as a nonlocal linear canonical transformation. All together $P_{l, m}, M_{\mu \nu}$ give the infinite vector representation of the BMS group. Note that the appearance of the BMS symmetry introduced for the scalar field [15] is not an asymptotic gauge symmetry as in [1]; it is a nonlocal symmetry of the free Klein-Gordon equation of motion.

We will construct a BMS invariant scalar product for the generalized momenta $w_{l, m}$, or for a rescaling of them. We write this product in terms of an infinite-dimensional matrix $\eta^{l, m ; l^{\prime}, m^{\prime}}$ that generalizes the Minkowski metric $\eta^{\mu \nu}$ for the scalar product of 4D momenta $k_{\mu}$. In a suitable basis of these zero modes $\eta^{l^{\prime}, m^{\prime} ; l, m}=\delta^{l^{\prime}, l} \delta^{m^{\prime}, m}$. The convergence of this scalar product has also been studied. ${ }^{1}$ The scalar product is the key ingredient for the definition of a configuration space (see, for example [17]), and therefore give meaning to coordinates $x^{l . m}$ conjugated to $w_{l, m}$.

Using this infinite-dimensional metric we will construct a quadratic Casimir with the supertranslations " $P^{2} "=\eta^{l, m ; l^{\prime}, m^{\prime}} P_{l, m}^{*} P_{l^{\prime}, m^{\prime}}$. Physically this Casimir allows us to define the BMS mass-shell constraint.

Our analysis of the scalar product and the Casimir " $P$ " " will be useful to study BMS symmetries and it could be useful to the study of the scattering $\mathrm{S}$ matrix, since the in-and-out states are free fields, and also to study particles and strings with BMS symmetries. The canonical realization of BMS considered in [15] and here could be

${ }^{1}$ Details and proof of the results will be presented elsewhere. 
extended to other fields with nonvanishing spin and to massless fields.

\section{CANONICAL REALIZATION OF BMS SYMMETRY}

We consider a free real scalar field $\Phi(t, \vec{x})$ of mass $m$ in four dimensions. The Fourier expansion is given by

$$
\Phi(t, \vec{x})=\int_{R^{3}} \tilde{d} k\left[a(\vec{k}) e^{-i(x \cdot k)}+a^{*}(\vec{k}) e^{i(x \cdot k)}\right]
$$

where $(\cdot)$ is the usual Minkowski Lorentz invariant product and $\tilde{d} k=\frac{d^{3} k}{\Omega(\vec{k})}, \quad \Omega(\vec{k})=(2 \pi)^{3} 2 \omega(\vec{k})=(2 \pi)^{3} 2 \sqrt{\vec{k}^{2}+m^{2}}$ and $k_{i}=-k^{i}$.

The realization of the Poincare group in terms of Fourier modes of the scalar field $\Phi(t, \vec{x})$ is given by

$$
\begin{gathered}
P_{\mu}=\int_{R^{3}} \tilde{d} k k_{\mu} a^{*}(\vec{k}) a(\vec{k}), \\
M^{0 j}=-i \int_{R^{3}} \tilde{d} k a^{*}(\vec{k}) \omega(\vec{k}) \frac{\partial}{\partial \vec{k}_{j}} a(\vec{k}), \\
M^{i j}=-i \int_{R^{3}} \tilde{d} k a^{*}(\vec{k})\left(k^{i} \frac{\partial}{\partial k^{j}}-k^{j} \frac{\partial}{\partial k^{i}}\right) a(\vec{k})
\end{gathered}
$$

as one checks the algebra by using the Poisson brackets $\left\{a(\vec{k}), a^{*}\left(\vec{k}^{\prime}\right)\right\}=-i \Omega(\vec{k}) \delta^{3}\left(\vec{k}-\vec{k}^{\prime}\right)$.

The mass-shell condition for $\Phi(t, \vec{x})$ is given by $q^{2}-m^{2}=0$; it defines the 3D spacelike hyperboloid, $H_{3}^{1}$, in the space of momenta, with coordinates $\vec{k}$. The relation with the embedding momenta $q_{\mu}$ is $q_{0}=\sqrt{\vec{k}^{2}+m^{2}}, q_{i}=k_{i}$. The induced metric on $H_{3}^{1}$ is given by

$$
d s^{2}=\hat{\eta}=\left(\frac{1}{\omega^{2}(\vec{k})} k_{i} k_{j}-\delta_{i j}\right) d k_{i} d k_{j}
$$

In polar coordinates $d s^{2}=-m^{2}\left(\frac{1}{r^{2}+1} d r^{2}+r^{2} d s_{S^{2}}^{2}\right)$, where $r=\frac{|\vec{k}|}{m}$, and $d s_{S^{2}}^{2}$ is the metric of the sphere.

This expression, apart dimensions, is used in [11] to give a foliation of the Minkowski space-time.

The 3D spacelike hyperboloid $H_{3}^{1}$ has constant negative curvature $R=-\frac{1}{m}$ [16]. It is a Euclidean Anti-de Sitter space $\mathrm{AdS}_{3}$ that can be written as the coset $\frac{S O(3,1)}{S O(2,1)}$. The coordinates are a global parametrization of the Euclidean $\mathrm{AdS}_{3}$ space. The Laplace-Beltrami operator on $H_{3}^{1}$ is given by

$$
\Delta=\vec{\nabla} \cdot \vec{\nabla}+\frac{2}{m^{2}} \vec{k} \cdot \vec{\nabla}+\frac{1}{m^{2}}(\vec{k} \cdot \vec{\nabla})^{2} .
$$

It is an elliptic operator and has the property $\Delta k_{\mu}=\frac{3}{m^{2}} k_{\mu}$. We introduce the operator $D$,

$$
D=-m^{2} \Delta+3
$$

the four momenta are zero modes of $D, D k^{\mu}=0$.

For a massive free Klein-Gordon in $2+1$ dimensions the corresponding operator is $D=-m^{2} \Delta_{2}+2$, where $\Delta_{2}$ is the Laplace-Beltrami in $H_{2}^{1}$.

The massless case can be studied taking the massless limit of the operator $D$, i.e.,

$$
D_{m=0}=-2 \vec{k} \cdot \vec{\Delta}-(\vec{k} \cdot \vec{\Delta})^{2}+3 .
$$

Since we want to find a generalization of four momenta to construct the supertranslations this property suggests that we study all zero modes of $D f(k)=0$. Introducing spherical coordinates, these are given by the functions [15]

$$
\begin{gathered}
w_{l, m}(\vec{k})=u_{l}(r) Y_{l, m}(\hat{k}), \\
u_{l}(r)=r^{l} F\left((l-1) / 2,(l+3) / 2 ; l+3 / 2 ;-r^{2}\right),
\end{gathered}
$$

where $F$ is the hypergeometric function, ${ }_{2} F_{1}, Y_{l, m}$ are the spherical harmonics and $r=\frac{|\vec{k}|}{m}$. The set $\left\{w_{l, m}\right\}$ is not the only set of solutions of the equation $\operatorname{Df}(\vec{k})=0$. For given values of $\{l, m\}$ there are two independent solutions of the equation. The first is $\left\{w_{l, m}\right\}$, which has a good behavior for $r \rightarrow 0$; the second set of solutions is singular in $r=0$. This is the reason for the choice $\left\{w_{l, m}\right\}$.

The functions $w_{l, m}$ span an infinite-dimensional nonunitary representation of the Lorentz group. This representation has the following properties:

(i) it is the only representation [15] with an invariant subspace of dimension four;

(ii) for $l=0,1 w_{l, m}$ is the four-vector $k^{\mu}$ in the spherical basis

$$
\begin{gathered}
w_{0,0}=\sqrt{1-r^{2}} Y_{0,0}=\frac{\omega(\vec{k})}{m} Y_{0,0}, \\
w_{1, m}=r Y_{1, m}=\frac{|\vec{k}|}{m} Y_{1, m} ;
\end{gathered}
$$

(iii) the functions $w_{l m}(r, \theta, \phi)$ have an asymptotic behavior like $k^{\mu}$, for all values of $l=0,1,2, \ldots$.

The presence of the zero modes $w_{l, m}(\vec{k})$ enables us to define the supertranslations in terms of Fourier modes 


$$
P_{l, m}=\int_{R^{3}} \tilde{d} k w_{l, m}(\vec{k}) a^{*}(\vec{k}) a(\vec{k}) .
$$

In Ref. [15] it is proven that these integrals are well defined and that $M^{\mu \nu}, P_{l, m}$ verify the BMS algebra.

\section{BMS INVARIANT SCALAR PRODUCT AND QUADRATIC CASIMIR}

Since we have an infinite set of function $w_{l, m}$ which generalizes the four momenta $k_{\mu}$, it is natural to ask if there exists a scalar product, invariant under BMS, which generalizes the usual Minkowski metric.

The strategy to construct this scalar product will be to require the Hermiticity of Lorenz generators acting on the set $w_{l, m}$ or a rescaling of them. We first consider the boost $K_{3}=M_{03}$. We look for a new basis of zero modes $\left\{k_{l, m}\right\}$ where the Hermiticity is studied using the diagonal scalar product for $l>1$,

$$
p\left(\vec{k}, \vec{k}^{\prime}\right)=\sum_{l>1} k_{l^{\prime}, m^{\prime}}^{*}(\vec{k}) \eta^{l^{\prime}, m^{\prime} ; l . m} k_{l m}\left(\vec{k}^{\prime}\right)
$$

with

$$
\eta^{l^{\prime}, m^{\prime} ; l . m}=\delta^{l^{\prime}, l} \delta^{m^{\prime}, m}
$$

For $l=0,1$ we will use the ordinary Minkowski metric.

The functions $\left\{k_{l, m}\right\}$ will be obtained by a rescaling of $\left\{w_{l, m}\right\}$,

$$
k_{l, m}=N(l) \frac{1}{M(l)} w_{l, m}, \quad M(l)=\frac{\Gamma(2) \Gamma\left(l+\frac{3}{2}\right)}{\Gamma\left(2+\frac{l}{2}\right) \Gamma\left(\frac{l+3}{2}\right)} .
$$

The factor $M(l)$ is introduced in order to have simple behavior for $l \rightarrow \infty$ since we have $\left|w_{l, m}\right| \leq$ $\sqrt{\frac{2 l+1}{4 \pi}} \sqrt{1+r^{2}} M(l)$.

The factor $N(l)$ is unknown and can be determined by imposing the Hermiticity of $K_{3}$. We have

$$
\begin{aligned}
K_{3} k_{l, m} & =\frac{N(l)}{N(l+1)} a_{l, m} k_{l+1, m}+\frac{N(l)}{N(l-1)} b_{l, m} k_{l-1, m} \\
& \equiv \mathcal{A}_{l, m} k_{l+1, m}+\mathcal{B}_{l, m} k_{l-1, m}
\end{aligned}
$$

where $a_{l, m}=-i(l-1) \mathcal{C}_{l+1, m}, b_{l, m}=i(l+2) \mathcal{C}_{l, m}$ and $\mathcal{C}_{l, m}=$ $\sqrt{\frac{(l-m)(l+m)}{(2 l-1)(2 l+1)}}$.

The generator $K_{3}$ will be self-adjoint if

$$
\mathcal{B}_{l, m}=\overline{A_{l-1, m}},
$$

which implies

$$
\left[i(l+2) \mathcal{C}_{l, m}\right] \frac{N(l)}{N(l-1)}=\left[i(l-2) \overline{\mathcal{C}}_{l, m}\right] \frac{N(l-1)}{N(l)} .
$$

If we define $E(l)=[N(l)]^{2}$, we get the recurrence relation

$$
E(l+1)=\frac{(l-1)}{(l+3)} E(l) .
$$

This recurrence equation is meaningful only for $l \geq 2$. The solution is given by

$$
E(l)=\frac{4 !}{(l+2)(l+1) l(l-1)} E(2), \quad l \geq 2,
$$

where $E(2)$ has an arbitrary value.

The action of $K_{3}$ on $\left\{k_{l, m}\right\}$, apart from a phase factor, is

$$
K_{3} k_{l, m}=\mathcal{A}_{l, m} k_{l+1, m}+\mathcal{B}_{l, m} k_{l-1, m},
$$

where

$$
\begin{aligned}
& \mathcal{A}_{l, m}=-i \sqrt{(l-1)(l+3)} \mathcal{C}_{l+1, m}, \\
& \mathcal{B}_{l, m}=i \sqrt{(l-2)(l+2)} \mathcal{C}_{l, m} .
\end{aligned}
$$

Note that

$$
\mathcal{A}_{l, m}=\overline{\mathcal{B}_{l+1, m}}, \quad \mathcal{A}_{1, m}=\mathcal{B}_{2, m}=0 .
$$

The action of the other generators can be obtained from $K_{1}=i\left[K_{3}, L_{2}\right], K_{2}=-i\left[K_{3}, L_{1}\right]$, and the standard action of $L_{3}$ and $L_{ \pm}$,

$$
\begin{gathered}
L_{3} Y_{l, m}=m Y_{l, m}, \\
L_{ \pm} Y_{l, m}=\sqrt{l(l+1)-m(m \pm 1)} Y_{l, m \pm 1} .
\end{gathered}
$$

We obtain

$$
K_{ \pm} k_{l, m}= \pm\left(\mathcal{D}_{l, m}^{ \pm} k_{l+1, m \pm 1}+\mathcal{E}_{l, m}^{ \pm} k_{l-1, m \pm 1}\right),
$$

where $\mathcal{A}$ and $\mathcal{B}$ are defined in Eq. (22) while $\mathcal{D}$ and $\mathcal{E}$ are

$$
\begin{aligned}
\mathcal{D}_{l, m}^{ \pm}= & \sqrt{l(l+1)-m(m \pm 1)} \mathcal{A}_{l, m \pm 1} \\
& -\sqrt{(l+1)(l+2)-m(m \pm 1)} \mathcal{A}_{l, m}
\end{aligned}
$$

and

$$
\begin{aligned}
\mathcal{E}_{l, m}^{ \pm}= & \sqrt{l(l+1)-m(m \pm 1)} \mathcal{B}_{l, m \pm 1} \\
& -\sqrt{(l-1)(l)-m(m \pm 1)} \mathcal{B}_{l, m} .
\end{aligned}
$$

The Hermiticity of the Lorentz generators implies the invariance of the scalar product under Lorentz 
JOAQUIM GOMIS and GIORGIO LONGHI

transformations. It is also invariant under supertranslations, since these form an Abelian algebra.

If we define the supertranslations using the set of zero modes $\left\{k_{l, m}\right\}$,

$$
P_{l, m}=\int_{R^{3}} \tilde{d} k k_{l, m}(\vec{k}) a^{*}(\vec{k}) a(\vec{k}) .
$$

The BMS algebra becomes

$$
\begin{aligned}
& {\left[M^{i, j}, P_{l, m}\right]=\epsilon_{i j k} P_{l^{\prime}, m^{\prime}}\left(L_{k}\right)_{l^{\prime}, m^{\prime} ; l, m},} \\
& {\left[M^{0, j}, P_{l, m}\right]=-P_{l^{\prime}, m^{\prime}}\left(K_{j}\right)_{l^{\prime}, m^{\prime} ; l, m},}
\end{aligned}
$$

where the matrices $\left\|L_{k}\right\|$ and $\left\|K_{j}\right\|$ are defined in Eqs. (22) and (24)-(26). This representation gives the vector representation of the BMS group. Let us observe that the supertranslations act as a canonical, but nonlocal, transformation of the scalar field and its momentum; see [15].

The invariant scalar product allows us to define a quadratic Casimir for the supertranslations
PHYSICAL REVIEW D 93, 025030 (2016)

$$
\text { " } P^{2} "=\eta^{l, m ; l^{\prime}, m^{\prime}} P_{l, m}^{*} P_{l^{\prime}, m^{\prime}} .
$$

One can check [" $P^{2}$ ", BMS $]=0$ using (30).

Therefore the BMS mass-shell condition is given by

$$
\eta^{l, m ; l^{\prime}, m^{\prime}} P_{l, m}^{*} P_{l^{\prime}, m^{\prime}}=m_{\mathrm{BMS}}^{2} .
$$

This condition will be useful to construct models for particles and strings with BMS symmetries.

\section{ACKNOWLEDGMENTS}

We acknowledge discussions with Roberto Casalbuoni and Jaume Gomis. This work has been supported in part by Grants No. FPA2013- 46570 (MICINN) and No. 2014SGR-104(Generalitat de Catalunya) and Consolider CPAN and by the Spanish MINECO under Project No. MDM2014-0369 of ICCUB (Unidad de Excelencia Maria de Maeztu).
[1] H. Bondi, M. G. J. van der Burg, and A. W. K. Metzner, Proc. R. Soc. A 269, 21 (1962); R. Sachs, Proc. R. Soc. A 270, 103 (1962); Phys. Rev. 128, 2851 (1962).

[2] A. Strominger, J. High Energy Phys. 07 (2014) 152.

[3] S. Weinberg, Phys. Rev. 140, B516 (1965).

[4] T. He, V. Lysov, P. Mitra, and A. Strominger, J. High Energy Phys. 05 (2015) 151.

[5] A. Strominger and A. Zhiboedov, arXiv:1411.5745.

[6] T. Banks, arXiv:hep-th/0306074.

[7] J. de Boer and S. N. Solodukhin, Nucl. Phys. B665, 545 (2003).

[8] G. Arcioni and C. Dappiaggi, Nucl. Phys. B674, 553 (2003).

[9] G. Barnich and C. Troessaert, J. High Energy Phys. 05 (2010) 062.
[10] T. He, P. Mitra, A. P. Porfyriadis, and A. Strominger, J. High Energy Phys. 10 (2014) 112.

[11] M. Campiglia and A. Laddha, J. High Energy Phys. 07 (2015) 115.

[12] D. Kapec, M. Pate, and A. Strominger, arXiv:1506.02906.

[13] A. Strominger, Talk and Strings 2015, https://strings 2015 .icts.res.in/.

[14] S. W. Hawking, arXiv:1509.01147.

[15] G. Longhi and M. Materassi, J. Math. Phys. (N.Y.) 40, 480 (1999).

[16] R. Raczka, N. Limic, and J. Niederle, J. Math. Phys. (N.Y.) 7, 1861 (1966); 7, 2026 (1966); 8, 1079 (1967).

[17] U. H. Niederer and L. O'Raifeartaigh, Fortschr. Phys. 22, 111 (1974). 